\title{
O Estímulo à Criatividade em Programas de Pós-Graduação segundo seus Estudantes
}

Eunice M. L. Soriano de Alencar ${ }^{12}$

Universidade Católica de Brasilia

\begin{abstract}
Resumo
O estudo investigou a extensão em que professores de pós-graduação implementam práticas que favorecem a criatividade, segundo pós-graduandos, e a avaliação destes quanto ao nível de sua criatividade, de seus professores e colegas, comparando-se os resultados com outros obtidos anteriormente com estudantes de graduação. Participaram do estudo 92 pós-graduandos que responderam a um inventário de incentivo à criatividade por parte de seus professores, e avaliaram o nível de sua criatividade, de seus professores e colegas. Observou-se maior incentivo a distintos fatores que se associam à criatividade segundo os pósgraduandos. Estes se perceberam ainda como mais criativos que os seus professores e colegas, considerando-se também e a seus professores como mais criativos do que estudantes de graduação. As condiç̃es mais favoráveis à criatividade na pós-graduação se devem possivelmente aos objetivos da mesma de enfatizar a produção do conhecimento.

Palavras-chave: Criatividade; pós-graduação; ensino.
\end{abstract}

The Incentive to Creativity in Graduate Programs according to their Students

Abstract

The study investigated the degree to which graduate professors implement instructional activities that foster creativity, according to graduate students, as well as these students' evaluation of their own level of creativity, their teachers' and their colleagues' level of creativity, comparing the results with others obtained with undergraduate students. Ninety-two graduate students answered an inventory of incentive to creativity and evaluated their own level of creativity, their teachers' and colleagues' level of creativity. More incentive to different factors which associate with creativity was pointed by the graduate students, comparing to the undergraduate ones. Graduate students judged themselves as more creative than their professors and colleagues. Moreover, they evaluate themselves and their professors as more creative than did the undergraduate students. The conditions more favorable to creativity in the graduate courses are due possibly to the goals of the graduate courses related to the production of knowledge.

Keywords: Creativity; graduate courses; teaching.

A maior parte das pesquisas sobre os ambientes educacionais e os seus efeitos nas habilidades criativas dos alunos tem focalizado os primeiros anos de escola. Inúmeros estudos foram desenvolvidos nos Estados Unidos por Torrance $(1965,1972,1979,1987,1993)$, Treffinger (1980, 1986, 1987, 1993; Treffinger, Isaksen \& Firestien, 1983) e Renzulli (1992), e no Brasil por Alencar (1974, 1975, 1984, 1985, 1990, 1991, 1993, 1994, 1996a, 1996b; Alencar \& Fleith, 1987; Alencar, Fleith, Shimabukuro \& Nobre, 1987; Fleith \& Alencar, 1992; Virgolim \& Alencar, 1993) e Wechsler (1985, 1987, 1993) sobre diferentes aspectos relacionados à criatividade, como habilidades de pensamento criativo, traços de

${ }^{1}$ Endereço para correspondência: Programa de Mestrado em Educação; Universidade Católica de Braślia, SGAN 916 Módulo B, Asa Norte, 70790160 Brasilia, DF. Fone: (61) 3405550, r. 155. E-mail: ealencar@pos.ucb.br ${ }^{2} \mathrm{~A}$ autora agradece ao $\mathrm{CNPq}$ pelo apoio recebido, à Profa. Maria de Fátima Bruno-Faria pela assistência na análise de dados, à Profa. Denise de Souza Fleith e a um revisor anônimo pela leitura e comentários críticos sobre o manuscrito personalidade de alunos mais criativos, características de professores que favorecem à criatividade, distintos procedimentos para facilitar o desenvolvimento e expressão das habilidades criativas, especialmente de estudantes do ensino fundamental. Estes e outros autores, como Isaksen e Murdock (1993), Sternberg (1991), IashinShaw (1994), têm chamado a atenção para a importância crucial de se estimular, desenvolver e oferecer práticas efetivas que levem a uma maior expressão do potencial criador. Lembram estes autores as características de nossa época, marcada pela incerteza, pela complexidade, e por mudanças profundas e intensas, que vêm ocorrendo em um ritmo cada vez mais acelerado, gerando continuamente novos problemas e desafios, que demandam soluções criativas.

Uma análise da literatura na área de criatividade indica ainda que, embora os anos de formação universitária, tanto de graduação como de pós-graduação, sejam da maior relevância em prover condições para que os estudantes se tornem conscientes de suas habilidades criativas e aprendam a fazer um uso maior de suas 
potencialidades criativas, muito pouca atenção tem sido dada ao desenvolvimento e cultivo das habilidades criativas no contexto universitário da maior parte dos países. Tanto Tolliver (1985) como Paulovich (1993) fazem críticas severas à educação universitária por não encorajar e mesmo reprimir o desenvolvimento e expressão das habilidades criativas dos estudantes. Também Toren (1993) refere-se à cultura de aprendizagem predominante no sistema universitário, pouco propícia à expressão da capacidade de criar e ainda Cohen (1997) que, em artigo sobre a educação universitária em Singapura, menciona uma pesquisa feita pelo Ministério da Educação daquele país, apontando a necessidade de expandir os objetivos de ensino com vistas a preparar o aluno para fazer uso de seu potencial para criar.

Observa-se, entretanto, um número reduzido de pesquisas realizadas com estudantes e professore universitários. Alguns estudos sobre este tema foram desenvolvidos nos Estados Unidos por pesquisadores interessados em examinar as propostas para desenvolvimento de habilidades criativas em cursos oferecidos por universidades norte-americanas e os seus efeitos sobre as habilidades criativas de estudantes universitários. Shallcross e Gawienowski (1989), por exemplo, descreveram um simpósio sobre criatividade levado a efeito na Universidade de Massachusetts, em 1986, para chamar a atenção para a importância da criatividade no contexto universitário e maneiras que poderiam ser utilizadas para se cultivar a criatividade naquele campus. Ainda nos Estados Unidos, um número significativo de pesquisas foi feito por Parnes e colaboradores, com amostras de estudantes universitários, com vistas a investigar tanto os efeitos de um programa semestral na estimulação deliberada da criatividade, como os efeitos nas habilidades criativas de cursos e de estratégias de resolução criativa de problemas (Parnes, 1987).

Um outro tópico que também já foi objeto de investigação diz respeito aos estilos de ensinar que promovem ou inibem altos níveis de produtividade criativa em estudantes universitários. Esta questão foi abordada previamente por Chambers (1973), em um estudo sobre o perfil de professores universitários que facilitavam o desenvolvimento de habilidades criativas dos estudantes, bem como daqueles que inibiam tal desenvolvimento. Este autor constatou que professores universitários que cultivam a criatividade nos seus cursos apresentam como características o entusiasmo, o encorajamento da independência por parte de seus alunos, a aceitação dos estudantes como iguais, o reforço do comportamento criativo dos estudantes e a condução das aulas de uma maneira mais informal. Por outro lado, os professores que inibem à criatividade foram descritos por seus alunos como mais preocupados com a memorização de conteúdos, com pouca originalidade e criatividade nas suas práticas de ensino, além de não enfatizarem o estudo independente e serem sarcásticos nas suas relações com os alunos. No Brasil, em um estudo prévio realizado por Rosas (1988), constatou-se falta de incentivo à criatividade nos cursos universitários, lembrando a autora a necessidade de o professor universitário ultrapassar o papel de conservador e transmissor, para o de inovador, produtor e criador. Entretanto, nenhum dado empírico é apresentado por Rosas para dar apoio a esta posição.

Além de um reduzido número de estudos sobre criatividade em cursos universitários, observou-se, através da revisão de literatura, que variáveis como gênero, curso e atividades desenvolvidas pelos universitários, não vêm sendo objeto de investigação.

Interessados neste tema, iniciamos em 1992, uma linha de pesquisa que incluiu, em um primeiro momento, a construção e validação de um inventário para avaliar a extensão em que diferentes aspectos relacionados à criatividades têm sido estimulados por professores universitários (Alencar, 1995, 1997). Outros aspectos investigados diziam respeito às habilidades de pensamento criativo de estudantes de graduação bem como a percepção destes sujeitos a respeito de suas próprias habilidades criativas, a de seus colegas e professores (Alencar, 1996b). Um dos fatores que nos levou ao desenvolvimento de tal linha de pesquisa foi a inexistência de estudos no Brasil sobre a extensão em que as habilidades criativas de estudantes universitários têm sido estimuladas por seus professores e também sobre a percepção destes estudantes sobre o nível de sua criatividade, de seus colegas e professores.

Os dados obtidos (Alencar, 1997), em um amostra de 428 estudantes de universidade pública e particular, das áreas de Ciências Humanas e Exatas, indicaram que, na opinião da amostra de universitários que participou do estudo, pouco incentivo têm dado os seus professores ao desenvolvimento da criatividade. Observou-se também que foram os estudantes dos primeiros semestres os que avaliaram os seus professores como propiciando maior estímulo a diferentes aspectos relacionados à criatividade comparativamente aos dos últimos semestres. Um dado que nos chamou a atenção foi o baixo desempenho dos estudantes universitários, de modo geral, nos testes de pensamento criativo. O seu desempenho foi, por exemplo, inferior ao apresentado por estudantes do ensino médio que participaram de um estudo anterior de nossa autoria (Alencar, Collares, Dias \& Julião, 1993). No estudo, correlações significativas foram ainda 
observadas entre os resultados de testes de pensamento criativo e aqueles obtidos na avaliação de seu nível de criatividade por parte dos universitários, sendo constatado que os estudantes que tiveram desempenho superior nos testes de pensamento criativo se avaliaram como significativamente mais criativos do que os seus colegas que apresentaram desempenho inferior naqueles mesmos testes (Alencar, 1996b). Notou-se ainda que os alunos, independentemente do tipo de universidade, consideraram-se significativamente mais criativos do que os seus professores, os quais foram considerados, na sua grande maioria, como muito pouco ou pouco criativos.

Complementando o estudo anteriormente descrito com estudantes de graduação, desenvolvemos a presente pesquisa, com o objetivo de responder às seguintes questões:

Vêm implementando os professores de pós-graduação práticas que favorecem o desenvolvimento das capacidades criativas, segundo percepção dos pós-graduandos?

Há diferenças nesta percepção entre estudantes do sexo masculino e feminino, entre os que trabalham ou se dedicam apenas à pós-graduação, e ainda entre os dos cursos de Psicologia e Educação comparativamente aos de outros cursos?

Como avaliam os estudantes de pós-graduação o nível de suas próprias habilidades criativas, de seus colegas e professores?

Há diferenças nesta avaliação entre estudantes de pósgraduação do sexo masculino e feminino, que trabalham ou somente estudam, dos cursos de Psicologia e Educação comparativamente aos de outros cursos de pós-graduação?

Foi ainda objetivo do estudo comparar os dados obtidos com pós-graduandos com aqueles coletados anteriormente com estudantes de graduação.

\section{Método}

\section{Participantes}

A amostra foi constituída por 92 estudantes de pósgraduação de uma universidade pública, sendo 43 do sexo masculino e 49 do sexo feminino. Deste total, 37 (40,2\% informaram que apenas estudavam e $54(58,7 \%)$ que, paralelamente às suas atividades como aluno de pósgraduação, também trabalhavam (faltou esta informação por parte de um sujeito). A idade dos sujeitos variou de 22 a 57 anos, sendo a média 32,3 anos. Dentre os 92 pósgraduandos, 36 cursavam mestrado (ou doutorado) em Psicologia, 8 em Educação, 13 em Ciências da Saúde, 8 em Agronomia, 6 em Geologia, 5 em Física, 5 em Estatística, 3 em Ciências Políticas e 2 em Botânica (seis dos respondentes deixaram de informar o seu curso). Quarenta e nove (53,3\%) informaram que estavam cursando o seu primeiro ano de pós-graduação e os demais que estavam no terceiro semestre ou subseqüentes.

\section{Instrumento}

Para avaliar a percepção do estudante quanto ao grau de incentivo a diferentes aspectos da criatividade por parte de seus professores de pós-graduação, foi utilizado um instrumento construído e validado anteriormente por Alencar (1995) que continha 19 itens. Alguns exemplos de itens do instrumento são apresentados a seguir: Os professores de pós-graduação, de modo geral:

Levam o aluno a perceber e conhecer pontos de vista divergentes sobre o mesmo problema ou tema de estudo.

Incentivam os alunos a fazer questões relativas aos temas estudados.

Estimulam a curiosidade dos alunos através das tarefas propostas em suas disciplinas.

Dão tempo aos alunos para pensar e desenvolver idéias novas. Cultivam nos alunos o gosto pela descoberta e pela busca de novos conhecimentos.

Utilizam provas e avaliações que exigem do aluno apenas a reprodução de conteúdo dado em classe ou contido nos livrostextos (inverte-se a pontuação deste item).

Cada um dos itens é respondido em uma escala de cinco pontos que varia de "discordo plenamente" até "concordo plenamente". Além de responderem a este inventário, os sujeitos responderam também os seguintes itens relativos à percepção da própria criatividade, de seus colegas e professores:

Como você se classifica em uma escala de criatividade?

Como você classificaria o nível de criatividade de seus professores da pós-graduação?

Como você classifica o nível de criatividade de seus colegas do programa de pós-graduação?

Estes itens foram respondidos em uma escala de sete pontos, que variava de (1) nada criativo(s) a (7) extremamente criativo (s).

\section{Procedimentos}

Embora tivesse sido prevista a aplicação do instrumento, de forma coletiva (durante o período de aula), em função de várias dificuldades encontradas, o mesmo foi também aplicado individualmente. Isto se deu pelas seguintes razões: não-autorização por parte do professor para esta aplicação durante o período em que estava em sala de aula; tempo insuficiente alocado pelo professor para que os seus estudantes respondessem ao instrumento no período reservado para sua disciplina; dificuldade de acesso ao professor para que este autorizasse a coleta de dados junto a seus alunos; disciplinas que funcionavam em um sistema tutorial, como estudo dirigido e atividade de pesquisa; recusa dos estudantes em responder ao instrumento quando o tempo alocado era no final da 
aula, alegando que tinham outras tarefas obrigatórias, que não poderiam ser deixadas para depois.

Para aplicação de forma individual, contatos eram feitos especialmente nas salas reservadas para os estudantes de pós-graduação, ocasião em que se solicitava a sua colaboração e se marcava um horário e local para que o mesmo respondesse ao instrumento. Neste contato inicial, era apresentado para o sujeito o objetivo do estudo e uma breve descrição do instrumento.

\section{Resultados}

Serão apresentados inicialmente os dados obtidos através do uso da escala que avaliava o estímulo a distintos aspectos relativos à criatividade por parte dos professores de pós-graduação. A seguir, os dados referentes à avaliação do nível de criatividade pessoal por parte dos pós-graduandos e a sua avaliação do nível de criatividade de seus professores e colegas.

Percepção dos Estudantes de Pós-Graduação quanto ao Estímulo à Criatividade por parte de seus Professores

$\mathrm{Na}$ Tabela 1, são apresentados a média, desvio padrão, valor $t$ e nível de significância na escala que avaliava o incentivo à criatividade por parte dos professores de pósgraduação, considerando-se as variáveis, sexo, trabalho e curso. No caso desta última variável, em função da variedade de cursos e do número reduzido de sujeitos em alguns deles, agruparam-se os respondentes do programa de pós-graduação em Psicologia e Educação, comparando os seus resultados com aqueles apresentados pelos respondentes dos demais cursos.

Como apresentado nesta Tabela, diferenças significativas foram observadas apenas na variável "curso" $(t=1,95 ; p=0,05)$. Nota-se que a média apresentada pelos pós-graduandos de Psicologia e Educação na escala foi significativamente inferior àquela apresentada pelos estudantes dos demais cursos.

Além do "escore" total na escala, foram analisadas diferenças relativas aos itens individuais do instrumento utilizado, considerando-se cada uma das variáveis independentes. Isto no sentido de se identificar aqueles itens em que diferenças significativas fossem observadas entre os diversos grupos.

Através desta análise, observou-se uma diferença significativa na variável "curso", a favor dos pósgraduandos dos demais cursos comparativamente aos dos cursos de Psicologia e Educação nos seguintes itens: Estimulam a iniciativa dos alunos $-t=1,93 ; p=0,05$;

Estimulam os alunos a pensar idéias novas relativas ao conteúdo da matéria - $t=1,97 ; p=0,05$;

Valorizam as idéias originais de seus alunos - $t=2,65 ; p=0,01$; Dão tempo ao aluno para pensar e desenvolver idéias novas - $\mathrm{t}=$ 2,$31 ; p=0,02$;

Promovem a autoconfiança de seus alunos - $t=3,296 ; p=0,001$.

Com relação à variável "trabalho", constatou-se diferença significativa, a favor dos pós-graduandos que trabalhavam, em apenas um único item, a saber: valorizam as idéias de seus alunos $(t=2,30, p=0,019)$.

Não foram encontradas diferenças significativas em quaisquer dos itens entre os pós-graduandos do sexo masculino e feminino.

Comparando os dados coletados com a amostra de pós-graduandos com aquela de pesquisa anterior de nossa autoria (Alencar, 1997), com uma amostra de estudantes de graduação, constatamos que a amostra do presente estudo percebeu maior estímulo à criatividade por parte de seus professores do que os estudantes de graduação. Na Tabela 2, são apresentados a média e o desvio padrão

Tabela 1. Média, Desvio Padrão, Valor $t$ na Escala de Incentivo à Criatividade pelos Professores de Pós-Graduação, segundo às Variáveis Sexo, Trabalho e Curso

\begin{tabular}{lllll}
\hline Variáveis & Média & $d p$ & $t$ & $p$ \\
\hline Sexo & & & & \\
$\quad$ Masculino & 3,38 & 0,62 & 0,05 & n.s. \\
$\quad$ Feminino & 3,38 & 0,81 & & \\
$\begin{array}{l}\text { Trabalho } \\
\quad \text { Sim }\end{array}$ & 3,48 & 0,70 & 1,41 & n.s. \\
$\quad$ Não & 3,27 & 0,72 & & \\
Curso & & & \\
$\quad$ Psicologia e Educação & 3,23 & 0,76 & 1,95 & \\
$\quad$ Outros & 3,53 & 0,67 & & \\
\hline
\end{tabular}


obtidos na escala por parte da amostra de estudantes de pós-graduação e de graduação, considerando-se as variáveis sexo e trabalho. Esta Tabela apresenta também o valor $t$ obtido. Nota-se, pelos dados aí apresentados, que tanto os pós-graduandos do sexo masculino quanto
Através do teste $t$ de Student para análise das diferenças entre médias, observou-se que os pós-graduandos se avaliaram como significativamente mais criativos do que os seus professores $(t=3,33 ; p 0,01)$ e colegas $(t=2,29$ p 0,05). Não foi, entretanto, significativa a diferença entre

Tabela 2. Média, Desvio Padrão, Valor $t$ na Escala de Incentivo à Criatividade (Estudantes de Graduação e de Pós-Graduação)

\begin{tabular}{lcccc}
\hline Variáveis & Média & $d p$ & $t$ & $p$ \\
\hline $\begin{array}{l}\text { Sexo masculino } \\
\text { graduação }\end{array}$ & 2,97 & 0,61 & 3,87 & 0,001 \\
pós-graduação & 3,38 & 0,62 & & \\
& & & & \\
Sexo feminino & 2,91 & 0,56 & 3,89 & 0,001 \\
graduação & 3,38 & 0,81 & & \\
pós-graduação & & & & \\
& 2,89 & 0,58 & 5,78 & 0,001 \\
Trabalho (sim) & 3,48 & 0,70 & & \\
graduação & & & & \\
pós-graduação & 3,01 & 0,58 & 2,04 & 0,05 \\
& 3,27 & 0,72 & & \\
$\begin{array}{l}\text { Trabalho (não) } \\
\text { graduação } \\
\text { pós-graduação }\end{array}$ & & & & \\
& & & & \\
\hline
\end{tabular}

os do sexo feminino, e ainda os pós-graduandos que trabalhavam ou apenas estudavam, obtiveram médias significativamente superiores àquelas obtidas pelos estudantes de graduação.

Avaliação do Nivel de Criatividade Pessoal e do Nivel de Criatividade de seus Professores e Colegas por parte dos PósGraduandos

$\mathrm{Na}$ Tabela 3, são apresentados a média e desviopadrão obtidos pelos sujeitos nas escalas que avaliavam o seu nível de criatividade, o de seus professores e colegas. a avaliação feita do nível de criatividade de seus professores e colegas $(t=1,25$; n. s. $)$

Compararam-se também os dados referentes a esta avaliação pelos pós-graduandos com outros similares obtidos anteriormente junto a uma amostra de estudantes de cursos de graduação. Neste sentido, apresentamos na Tabela 4, a média e o desvio padrão obtidos nesta avaliação em uma amostra de 428 universitários de duas instituições de ensino superior, sendo uma pública e outra particular.

Tabela 3. Média e Desvio Padrão na Avaliação do seu Nível de Criatividade, do de seus Colegas e Professores por parte dos Pós-Graduandos

\begin{tabular}{lcc}
\hline Avaliação do nível de criatividade & $m$ & $d p$ \\
\hline De si mesmo & 4,23 & 0,85 \\
De seus professores & 3,74 & 1,12 \\
De seus colegas & 3,93 & 0,90 \\
\end{tabular}


Tabela 4. Média e Desvio Padrão na Avaliação do seu Nível de Criatividade, do de seus Professores e Colegas por parte de Universitários

\begin{tabular}{lcc}
\hline Avaliação & $m$ & $d p$ \\
\hline De si mesmo & 3,65 & 1,20 \\
De seus professores & 2,87 & 1,11 \\
De seus colegas & 3,73 & 1,12 \\
\hline
\end{tabular}

Comparando-se a média apresentada na autoavaliação por parte dos estudantes de graduação e pósgraduação, observou-se que os pós-graduandos se consideraram como significativamente mais criativos do que os estudantes de graduação $(t=5,47 ; p=0,001)$. Observou-se ainda que os pós-graduandos consideraram os seus professores como significativamente mais criativos do que os estudantes de graduação $(t=6,69 ; p=0,001)$. Entretanto, não foi observada diferença significativa entre a avaliação feita de seus colegas por parte de estudantes de graduação e de pós-graduação $(t=1,80$; n.s. $)$.

\section{Discussão}

O presente estudo teve como um de seus objetivos principais investigar a percepção de pós-graduandos quanto ao estímulo à criatividade por parte de seus professores. Diferindo de dados obtidos com relação a este aspecto junto a uma amostra de estudantes de graduação, constatou-se que os pós-graduandos consideraram os seus professores como apresentando maior incentivo a distintos aspectos que favorecem à expressão da criatividade. Nota-se que a escala utilizada incluía itens referentes a traços de personalidade - como autoconfiança, iniciativa e independência; outros relativos a um ambiente propício à produção de novas idéias; ainda outros a metodologias de ensino e tempo proporcionado ao aluno para pensar e desenvolver idéias novas. Práticas docentes que influenciam positivamente as habilidades criativas foram percebidas pelos participantes do presente estudo.

Esta percepção de maior estímulo à criatividade por parte dos professores de pós-graduação possivelmente pode ser explicada pelas melhores condições oferecidas ao aluno para a sua formação profissional e como pesquisador nos cursos de pós graduação stricto sensu. Sabe-se que cultivar o gosto pela descoberta e pela busca de novos conhecimentos, motivar os alunos a pensar e a raciocinar, estimular a produção de novas idéias, desenvolver habilidades de análise crítica e valorizar as idéias dos alunos certamente contribuem para a formação do pesquisador. Esta formação é um dos objetivos centrais de qualquer programa de pós-graduação.

Observa-se também um número mais reduzido de estudantes por turmas nas grande maioria de disciplinas oferecidas nos programas de mestrado e doutorado comparativamente àquelas da graduação. Este é um fator que também pode facilitar ao professor promover condições mais adequadas ao desenvolvimento das habilidades criativas, o que foi percebido pelos respondentes.

Ao analisar as respostas dos pós-graduandos na escala que avaliava o incentivo à criatividade, considerando-se as variáveis sexo, trabalho e curso (Psicologia e Educação, comparativamente aos demais), constatou-se que apenas na variável "curso", diferença significativa foi observada. Entretanto, contrário às nossas expectativas, foram os pós-graduandos dos cursos de Psicologia e Educação, comparativamente aos pós-graduandos dos demais cursos, os que perceberam menor estímulo ao desenvolvimento e expressão das habilidades criativas. Este é um resultado difícil de ser explicado, especialmente porque considerávamos que os professores tanto da Psicologia como da Educação estivessem melhor equipados para prover um ambiente propício ao desenvolvimento e expressão da criatividade. Nota-se, porém, que a amostra de pós-graduandos de cada um dos cursos representados no presente estudo foi relativamente pequena. Por esta razão, considera-se que esta variável mereça ser melhor explorada em estudos futuros.

Constatou-se no presente estudo que os pósgraduandos se perceberam como significativamente mais criativos do que os seus professores e colegas. Este resultado possivelmente reflete experiências anteriores bem-sucedidas dos pós-graduandos, onde tiveram oportunidades de se expressar criativamente. E relevante destacar que pesquisas sobre a avaliação de habilidades criativas de estudantes têm utilizado tradicionalmente avaliações feitas por professores e colegas (Alencar, 1985; Hocevar \& Bachelor, 1989). Entretanto, nossas observações durante programas de criatividade em escolas indicaram que os alunos fazem julgamentos a respeito de suas competências e habilidades criativas, bem como as de seus professores e colegas, que influenciam e orientam seu comportamento e ações. Isto está de acordo com estudos prévios sobre o autoconceito, que focalizaram a extensão em que a percepção e avaliação do indivíduo sobre si mesmo orientam e controlam seu comportamento (Markus \& Wurf, 1987). Estes estudos sugerem que, se os indivíduos se percebem e se avaliam como competentes, capazes e criativos, são mais propensos a correr riscos, a sentir-se mais confiantes para 
expressar idéias e exibir comportamento criativo. No presente estudo, pode-se supor que a avaliação positiva de seu nível de criatividade possa estar se refletindo no seu desempenho e na realização de suas tarefas acadêmicas.

Ao compararmos os resultados apresentados pelos pós-graduandos, que fizeram parte do presente estudo com outros relativos a esta mesma avaliação, obtidos junto a estudantes de graduação (Alencar, 1996b), observamos diferenças altamente significativas a favor dos pósgraduandos. Estes consideraram-se a si mesmos e a seus professores como significativamente mais criativos do que os estudantes de graduação. Entretanto, não foi significativa a diferença entre a avaliação feita do nível de criatividade dos colegas entre os pós-graduandos e os estudantes de graduação.

Esta percepção de si mesmo como mais criativo do que a dos estudantes de graduação por parte dos pósgraduandos possivelmente pode ser explicada pelas exigências dos cursos de pós-graduação. Para serem bemsucedidos nestes cursos, os pós-graduandos necessitam apresentar projetos que denotem novas idéias e originalidade, havendo maior estímulo para que busquem novos conhecimentos e maior independência de pensamento, o que deve ter contribuído para que se percebessem como mais criativos. Por outro lado, nos cursos de graduação, há menos oportunidades para o aluno expressar as suas habilidades criativas em sala de aula, o que pode ter se refletido em sua auto-avaliação. Como apontam vários autores, dentre eles, Tolliver (1985), Paulovich (1993), Slabbert (1994) e Alencar (1997), um ambiente que não dê apoio à criatividade pode inibir ou reprimir as habilidades criativas do estudante, com influência na percepção do estudante de suas próprias habilidades criativas.

Os resultados obtidos no presente estudo apontam várias questões a serem exploradas em estudos futuros. Uma delas diz respeito à percepção de um maior número de pós-graduandos das distintas áreas do conhecimento quanto ao estímulo à criatividade nos programas em que estão inseridos, tecendo comparações entre as diversas amostras. Uma segunda diz respeito à percepção do estímulo à criatividade nos cursos de pós-graduação por parte de seus professores. Seria também relevante investigar o grau de concordância entre a percepção de professores e alunos de pós-graduação quanto ao estímulo à criatividade.

\section{Referências}

Alencar, E. M. L .S. (1974). Um estudo de criatividade. Arquivos Brasileiros de Psicologia Aplicada, 26(2), 59-69.
Alencar, E. M. L. S. (1975). Efeitos de um programa de criatividade em alunos de $4^{a}$ e $5^{a}$ séries. Arquivos Brasileiros de Psicologia Aplicada, 27(4), 3 15.

Alencar, E. M. L. S. (1984). Características psicossociais de crianças brasileiras mais e menos criativas. Interamerican Journal of Psychology, 18(1-2), 87-99. Alencar, E. M. L. S. (1985, julho/agosto). Creativity in Brazilian schools. Giffed, Creative, Talented, 39, 15-17.

Alencar, E. M. L. S. (1990). Training teachers to teach for creativity. European Journal for High Ability, 1(1-2), 222-226.

Alencar, E. M. L. S. (1991). O estímulo à criatividade no contexto educacional. Revista Portuguesa de Educação, 4(1), 111-117.

Alencar, E. M. L. S. (1993). Thinking in the future: The need to promote creativity in the educational context. Giffed Education International, 9(2), 93-96.

Alencar, E. M. L. S. (1994). Creativity in the Brazilian educational context: Two decades of research. Giffed and Talented International, 9(1), 4-7.

Alencar, E.M.L.S. (1995). Developing creative abilities at the university level. European Journal for High Ability, 6, 82-90.

Alencar, E. M. L. S. (1996a). La escuela y el desarrollo del talento creativo Ideación, 9, 12-16.

Alencar, E. M. L. S. (1996b). University students' evaluation of their own level of creativity and their teachers' and colleagues' level of creativity. Giffed Education International, 11, 128-130.

Alencar, E. M. L. S. (1997). O estímulo à criatividade no contexto universitário. Psicologia Escolar e Educacional, 1(2-3), 29-38.

Alencar, E. M. L. S., Collares, K., Dias, L. \& Julião, S. (1993). Efeitos a curto e médio prazos de um programa de treinamento de criatividade em estudantes do ensino de segundo grau [Resumos]. Sociedade Brasileira estudantes do ensino de segundo grau [Resumos]. Sociedade Brasileira
de Psicologia (Org.), Anais da XXIII Reunião Anual (p. 364). Ribeirão de Psicologia $(\mathrm{O}, \mathrm{P}$
Preto, SP: SBP.

Alencar, E. M. L. S. \& Fleith, D. S. (1987). Avaliação de um programa de criatividade por professores do ensino de primeiro grau. Forum Educacional, 11(1), 51-63.

Alencar, E. M. L. S., Fleith, D. S., Shimabukuro, L A. \& Nobre, M. A. (1987), Efeitos de um programa de treinamento de criatividade para professores do ensino de primeiro grau nas habilidades de professores do ensino de primeiro grau nas habilidades de
pensamento criativo do aluno. Interamerican Journal of Psychology, 21(1), 56-71.

Chambers, J. A. (1973). College teachers: Their effect on creativity of students. Journal of Educational Psychology, 65, 326-334.

Cohen, D. (1997, Setembro). Singapore wants its universities to encourage more creativity. The Chronicle of Higher Education, 71-72.

Fleith, D. S. \& Alencar, E. M. L. S. (1992). Efeitos de um programa de treinamento de criatividade em estudantes normalistas. Estudos de Psicologia, 9(2), 9-38.

Hocevar, D. \& Bachelor, P. (1989). A taxonomy and critique of measurements used in the study of creativity. Em J. A. Glover, R. R. Ronning \& C. R. Reynolds (Orgs.), Handbook of creativity (pp. 53-75). New York: Plenum Press.

Iashin-Shaw, I. (1994). Cognitive structures of creativity: Implications for instructional design. European Journal for High Ability, 5, 24-38.

Isaksen, S. G. \& Murdock, M. C. (1993). The emergence of a discipline: Issues and approaches to the study of creativity. Em S. G. Isaksen, M. C. Murdock, R. L. Firestien, \& D. J. Treffinger (Orgs.), Understanding and recognizing creativity). The emergence of a discipline (pp. 13-47). Norwood, NJ: Ablex.

Markus, H. \& Wurf, E. (1987). The dynamic self-concept: A social psychological perspective. Annual Review of Psychology, 38, 299-337.

Parnes, S. J. (1987). The Creative Studies Project. Em S. G. Isaksen (Org.), Frontiers of creativity researcb. Beyond the basics (pp. 156-188). Buffalo, NY: Bearly.

Paulovich, A. (1993). Creativity and graduate education. Molecular Biology of the Cell, 4, 565-568. 
Renzulli, J. S. (1992). A general theory for the development of creative productivity in young people. Em F. Monks \& W. Peters (Orgs), Talent for the future (pp. 51-72). Assen/Maastricht, The Netherlands: Van Gorcum

Rosas, A. (1988). Universidade e criatividade. Anais do VII Seminário Nacional sobre Superdotados (pp. 121-124). Rio de Janeiro: SENAI

Shallcross, D. J., \& Gawienowski, A. M. (1989). Top experts address issue on creativity gap in higher education. Journal of Creative Behavior, 23, 75-84.

Slabbert, J. A. (1994). Creativity in education revisited. Reflection in aid of progression. The Journal of Creative Behavior, 28, 6-69.

Sternberg. R. J. (1991, Julho). A theory of creativity. Trabalho apresentado na XIV ISPA Colloquium, Braga, Portugal.

Tolliver, J. M. (1985). Creativity at university. Giffed Education Inernational, 3 32-35.

Toren, K. (1993). Transformations in management education. American Behavior Scientist, 37, 112-120

Torrance, E. P. (1965). Rewarding creative behavior. Experiments in classroom creativity. Englewood Cliffs, NJ: Prentice Hall.

Torrance, E. P. (1972). Can we teach children to think creatively? Journal of Creative Behavior, 6, 114-143.

Torrance, E. P. (1979). The search for satory and creativity. Buffalo, NY: Bearly Limited.

Torrance, E. P. (1987). Teaching for creativity. Em S. G. Isaksen (Org.), Frontiers of creativity research. Beyond the basics (pp. 189-215). Buffalo, NY: Bearly Limited.

Torrance, E. P. (1993). Experiences in developing technology for creative education. Em S. G. Isaksen, M. C.Murdock, R. L. Firestien \& D. J.
Treffinger (Orgs.) Understanding and recognizing creativity. The emergence of Treffinger (Orgs.) Understanding and recognizing
a discipline (pp. 158-201). Norwood, NJ: Ablex.

reffinger, D. J. (1980). Encouraging creative learning for the gifted and talented. Ventura.: Ventura County School Superintendent's Office.
Treffinger, D. J. (1986). Research on creativity. Giffed Child Quarterly, 30(1), 15-19.

Treffinger, D. J. (1987). Research on creativity assessment Em S. G. Isaksen (Org.), Frontiers of creativity research (pp. 103-109). Buffalo, NY: Bearly. Treffinger, D. J. (1993). Stimulating creativity: Issues and future directions. Em S. G. Isaksen, M. C. Murdock, R. L. Firestien, \& D. J. Treffinger (Orgs.), Nurturing and developing creativity: The emergence of a discipline (pp. 8-30). Norwood, NJ: Ablex.

Treffinger. D. J. Isaksen, S. G. \& Firestien, R. L. (1983). Theoretical perspectives on creative learning and its facilitation: An overview. Journal of Creative Behavior, 17(1), 9-16.

Virgolim, A. M. R. \& Alencar, E. M. L. S. (1993). Habilidades de pensamento criativo entre estudantes de escolas tradicional, intermediária e aberta. Psicologia: Teoria e Pesquisa, 9, 601-610.

Wechsler, S. (1985). A identificacão do talento criativo nos Estados Unidos e no Brasil. Psicologia: Teoria e Pesquisa, 1, 14-17.

Wechsler, S. (1987). Efeitos do treinamento de criatividade em crianças bem-dotadas e regulares. Arquivos Brasileiros de Psicologia, 39, 95-111.

Wechsler, S. (1993). Issues on stimulating creativity in the schools: A South American perspective. Em S. G. Isaksen, M. C. Murdock, R. L. Firestien \& D. J. Treffinger (Orgs.), Nurturing and developing creativity: The emergence of a discipline (pp. 100-132). Norwood, NJ: Ablex.

Sobre a autora

Eunice Soriano de Alencar é Professora do Programa de Mestrado em Educação da Universidade

Católica de Brasília. Ph.D. em Psicologia pela Universidade de Purdue, USA. Pesquisadora do CNPq.

Vice-Presidente da Federação Ibero-Americana do Conselho Mundial para o Superdotado e Talentoso. 